\title{
A Practical Hull Form Design of Ferry Using Hybrid Scheme Method and Performing Experiment
}

\author{
Suandar Baso, Wardina Suwedy, Rosmani, Lukman Bochary and Andi Ardianti \\ Department of Naval Architecture, Faculty of Engineering,Hasanuddin University,Makassar 90245, Indonesia
}

\begin{abstract}
Prediction of ship performance in preliminary ship design is an important consideration. It could guarantee ship in safe and comfort. However, many design works did not involve simultaneously ship performances predictions in preliminary design. Moreover, ship designers sometimes modified a ship form to obtain proper design without ship performance consideration. Therefore, this study concerns on predictions of total resistance and added wave resistance of a ferry using a hybrid particle-grid method and then its motions response after modifying bow and stern parts by conducting experiment. Research results show total resistance and added wave resistance have a significant different, therefore, it would be an important consideration in determining ship powering in preliminary ship design. The non-dimensional added wave resistance increases in increasing wave length from $\lambda / L_{p p}=0.5$ to 1.0 and it decreases after $L / \lambda=1.0$. In addition, it tends to decrease caused by increasing ship speed. The comparison of averaged heave and pitch amplitudes between basic forms after modifying bow and stern parts is quiet similar. However, the rolling amplitude of the modified form is significantly higher comparing with the basic form. We conclude that a ship could be design in preliminary design take into account performances predictions by using numerical method and experimental work.
\end{abstract}

Keywords: Resistance,added wave resistance, heave motion, pitch motion, rolling motion.

\section{Introduction}

A proper ship design is ownership expectation and it could contribute some beneficial costs. In order to obtain a proper ship design, it is iteratively processed that reflects the design methodology and strategy. However, this would become difficult to use appropriate design tools and possible take longer time.

In 1946, CFD (computational fluid dynamics)methods began replacing analytic equations and experimental fluid dynamics in ship design. Over the past several years, according to rapid advances in hardware and several computational techniques, some researchers concern on developing CFD method take into account all hydrodynamic behaviors to become applicable and practical tools.

In naval architecture and ocean engineering field, the developed CFD methods have been done involved several computational techniques [1-5] developed The

Corresponding author: Suandar Baso, Ph.D., research field: naval architecture. E-mail: andar_baso@yahoo.co.id.
CFD method to apply to hull form design and sterns with devices of complex forms. However, the solutions are sufficient accurate. They still require experimental results.

Recently, we have developed hybrid scheme which is Eulerian grid with Lagrangian particles [6] to combine the advantages and to compensate for the disadvantages in both the grid-based and the particle based methods. This developed method has been applied to many various cases in naval architecture and ocean engineering fields. It was applied to ship propulsion performance [7], ship seakeeping performace [8], ship motions with hydroelastic effects [8], and investigation of resistance reduction by improving stern part [9], etc. However, we need more quantitative assessments in integrating design for preliminary design step purpose.

In additions, ship hull form under still water could be optimized to obtained proper design based on minimum resistance and good motions by computing simultaneously during preliminary ship design process. 
Therefore, in this study, the developed method has been extended its usefulness in preliminary design step combined with conducting experiment.

\section{Computational Method}

In this section, the numerical method, which combines the Eulerian scheme and the Lagrangian particles by coupling the SPH (Smoothed Particle Hydrodynamics) method and CIP(Cubic-Interpolated Pseudo Particle) method with particle, are described concisely. First, the CIP method with particles is introduced as a numerical scheme that combines the accuracy of Lagragian front tracking. Thereafter, the $\mathrm{SPH}$ method is employed to calculate deformation, strain, stress of elastic body, and 3D motion.

\subsection{Arrangement of Grids and Particles}

The developed Eulerian scheme with Lagrangian particles has been illustrated as shown in Fig.1. This scheme uses a staggered grid system and has two types of Lagrange particles, i.e. SPH particles todescribe solid and free surface particles to capture free surface accurately.

Density function $\phi_{I}$ defined on a grid node is corrected by using density function $\phi_{P}$ on free surface particles within referenced area with radius $h$. A smooth approximation of a density function can be

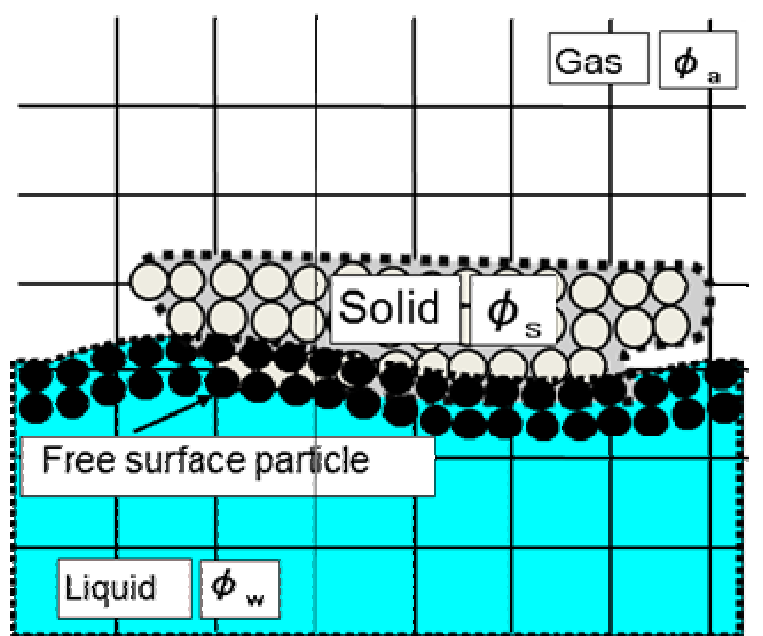

Fig. 1Illustration of the proposed model ( $\phi_{I}$ indicates density function; Lagrangian particles are located on Eulerian grid). constructed by using a Kernel function in the SPH method.

\subsection{Governing Equations for Fluid Phase}

The governing equations for fluid phase consist of the mass conservation equation, incompressible Navier-Stokes equation and the equation of continuity, $I$-phase density function $\phi_{I}\left(0 \leq \phi_{I} \leq 1\right)$ and its advection equation. The equations are expressed as follows:

$$
\begin{gathered}
\frac{\partial \bar{u}_{i}}{\partial x_{i}}=0 \ldots \ldots \ldots \ldots \\
\frac{\partial \bar{u}_{i}}{\partial t}+\bar{u}_{j} \frac{\partial \bar{u}_{i}}{\partial x_{j}}= \\
-\frac{1}{\rho} \frac{\partial \bar{P}}{\partial x_{i}}-\frac{\partial \tau_{i j}}{\partial x_{j}}+\frac{\mu}{\rho} \frac{\partial^{2} \bar{u}_{i}}{\partial x_{j} \partial x_{j}}+g_{i}+\bar{F}_{f s i} \\
\frac{\partial \varphi_{I}}{\partial t}+\bar{u}_{j} \frac{\partial \varphi_{I}}{\partial x_{j}}=0 \ldots \ldots
\end{gathered}
$$

where, $u_{i}$ is the velocity, $\mu$ the coefficient of fluid viscosity, $\rho$ the fluid density, $P$ the pressure, $F_{f s i}$ the fluid-structure interaction, $g_{i}$ the acceleration due to garvity, $\tau_{i j}$ the SGS stress term, and $\phi_{I}$ the density function. To reduce model parameters, the SGS (Subgrid-Scale)stress term is solved by using the dynamic SGS model proposed by Germano.More details are provided by Mutsuda and Yasuda [10].

\subsection{Advection Step and Non-advection Step}

The governing equations are solved by using the splitting method which is suitable for solving a multi-phase flow without smearing a density across interface between air and water. The advection step is calculated by the CIP method proposed by Takewaki and Yabe [11]. Then, the type-M scheme of the CIP method is employed by using the third-order accuracy in time and space. On the other hand, the non-advection step is solved by using the second-order finite difference method.

\subsection{Governing Equations for Solid Phase}

The governing equations for solid phase are the 
continuity and momentum equations as follows:

$$
\begin{array}{r}
\frac{\mathrm{D} \rho}{\mathrm{D} t}+\rho \frac{\partial u^{i}}{\partial x^{i}}=0 \\
\rho \frac{\mathrm{D} u^{i}}{\mathrm{D} t}=\frac{\partial \sigma^{i j}}{\partial x^{j}}+g^{i}-F_{f s i}^{i}
\end{array}
$$

where, $S^{i j}$ is the density, $u^{i}$ the velocity, $P=-\sigma_{k k} / 3$ the position vector of vector $j$ components, the stress tensor of the solid phase, and $F_{f s i}$ the fluid structure interaction term. The stress tensor $\sigma_{s}^{i j}$ in Eq.(5) is given by:

$$
\sigma_{s}^{i j}=-P \delta^{i j}+S^{i j}
$$

where, $S^{i j}$ is the deviatoric stress tensor, the pressure solved by the Poisson'sEq. (9) as mentioned below.

Our numerical model considers a large deformation of an elastoplastic body. The solid body changes at every calculation step by using the following equation:

$$
\left\{\mathrm{d} S^{i j}\right\}=\left[D^{e p}\right]\left\{\mathrm{d} \varepsilon^{i j}\right\}
$$

where, $D^{e p}$ is the elastoplastic matrix, $\mathrm{d} \varepsilon^{i j}$ the time increment of the strain, and $\mathrm{d} S^{i j}$ the time increment of the deviatoric stress. To solve rotation of the solid phase during a deformation, the Jaumann derivative is used to ensure material frame indifference with respect to the rotation as follow:

$$
\frac{\mathrm{d} S^{i j}}{\mathrm{~d} t}=2 \mu\left(\dot{\varepsilon}^{i j}-\frac{1}{3} \delta_{i j} \dot{\varepsilon}^{i j}\right)+S^{i k} \Omega^{j k}+\Omega^{i k} S^{k j}(8)
$$

where, $\dot{\varepsilon}$ is the strain rate tensor and $\Omega$ the spin tensor.

The pressure with specified jump conditions is solved by the Poisson's equation given by:

$$
\nabla \cdot\left(\frac{\nabla P^{n+1}}{\rho^{*}}\right)=\frac{\nabla \cdot u^{*}}{\Delta t}
$$

where, $*$ denotes a physical value after the advection step. The pressure for solid phase can be obtained by this equation and be applied in solving a solid deformation.

The fluid structure interaction $F_{f s i}$ is solved by acceleration obtained from the pressure on the SPH particles interpolated using the pressure on grids solved by the Poisson's Eq. (2). In the model, the fluid structure interaction $F_{f s i}$ in Eqs.(2) and (5) can be given by the following equation:

$$
F_{f s i}\left(\mathbf{r}_{a}\right)=-\frac{1}{\rho\left(\mathbf{r}_{a}\right)} \sum_{b} m_{b} \frac{P\left(\mathbf{r}_{b}\right)}{\rho\left(\mathbf{r}_{b}\right)} \nabla_{a} W\left(\mathbf{r}_{a}-\mathbf{r}_{b}, h\right)(10)
$$

To keep computational efficiency and stability, the time increment in the solid phase is approximately $1 / 10$ to $1 / 50$ of that in fluid phase.

\subsection{Ship Motions}

A ship motion is solved by using information obtained from SPH particles because a ship hull consists of SPH particles capturing motion and deformation of a ship. Therefore, the 3D motion of a ship hull is represented by describing translation and rotation of the center of gravity of a ship hull by using the following equations:

$$
\begin{gathered}
\frac{\partial^{2} x_{s, k}}{\partial t^{2}}=\frac{F_{s, k}}{m_{i}}-F_{f s i} \\
I \frac{\partial \omega_{i}}{\partial t}=T_{i} \\
\frac{\partial \theta_{i}}{\partial t}=\omega_{i}
\end{gathered}
$$

where, $\theta_{I}$ is the rotational angle, $\omega_{i}$ the angular velocity, $T_{i}$ the torque, $I$ the inertia moment, and $F_{f s i}$ the fluid structure interaction. In addition, the center of gravity of a ship hull can be obtained by solving the inertia moment of SPH particles, and this is calculated by using Baraff theory [12]. Therefore, the coordinates of velocity of each SPH particle in every time step can be tracked by using the rotation matrix and the amount of the angle rotation of the center of gravity. The quaternion is also used instead of the rotation matrix $R(t)$ in $3 \mathrm{D}$ to avoid the Gimbal lock phenomenon.

\section{Experimental Method}

The motions test is performed in water tank which belongs to Naval Architecture Department, Hasanuddin University. The purposes are to measure heave, pitch, and roll amplitudes in zero speed.

The ferry model was made of wood and it is 
suitable with dimensions of the water tank. The body lines plan of the ship is shown in Fig. 2. Then, the main dimensions of the actual ship and the model are presented in Table 1.

The experimental set up was determined and designed. The ship model is kept on constant and free position with still water draft. The main dimensions of water tank are provided in Table 2. The water tank is equipped with wave maker. Here, wave periode $T$ is set to $1,200 \mathrm{~ms}$. The circle of regular wave is stable greater than $60,000 \mathrm{~ms}$. Therefore, the experimental data is collected from $60,000 \mathrm{~ms}$ to $70,000 \mathrm{~ms}$. The experiment design and motions measurement are shown schematically in Fig. 3.

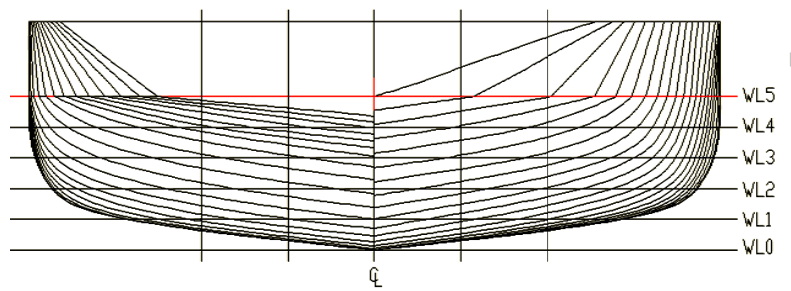

Fig. 2 Ship body lines plan.

Table 1 Main dimensions of the actual shipand the model.

\begin{tabular}{lll}
\hline & Actual ship & Model \\
\hline $\operatorname{Lpp}(\mathrm{m})$ & 47.1 & 0.60 \\
$B(\mathrm{~m})$ & 14 & 0.17 \\
$H(\mathrm{~m})$ & 3.35 & 0.05 \\
$T(\mathrm{~m})$ & 2.24 & 0.03 \\
$V(\mathrm{knot})$ & 12 & - \\
\hline
\end{tabular}

Table 2 The dimensions of the water tank.

\begin{tabular}{ll}
\hline Length $(\mathrm{m})$ & 18 \\
\hline Width $(\mathrm{m})$ & 1.35 \\
Height $(\mathrm{m})$ & 1.2 \\
Depth $(\mathrm{m})$ & 0.8 \\
\hline
\end{tabular}

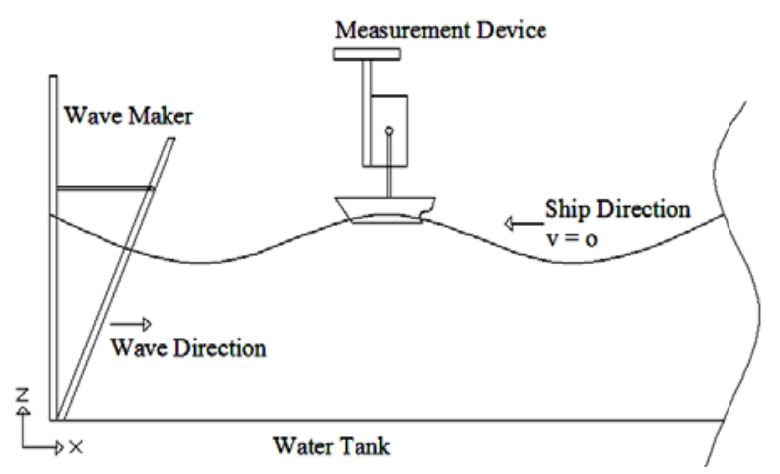

Fig. 3 Experiment design.

\section{Validations}

Fig. 4 shows comparison results of resistance of high speed ferry in calm water between computation and experiment. From the figure, the tendency of the resistance shows the same. Then, this shows overall results of computation are in good agreement with experimental results.

Figs. 5 and 6 show comparison of fishing boat motions between numerical and experimental results.

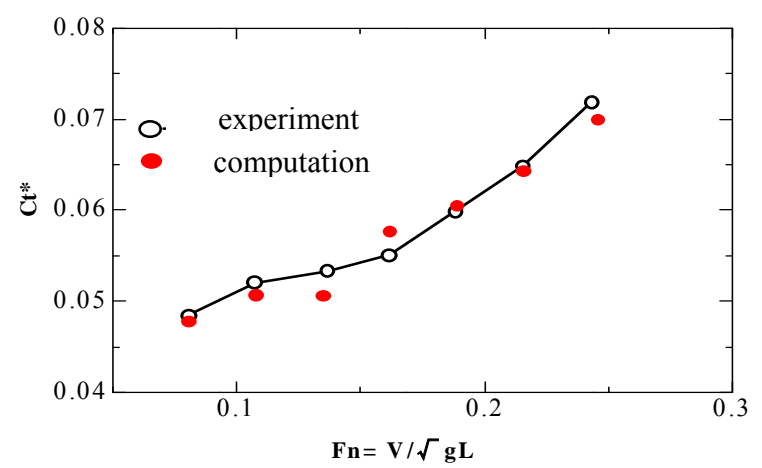

Fig. 4 Comparison of the resistance between computation and experiment.

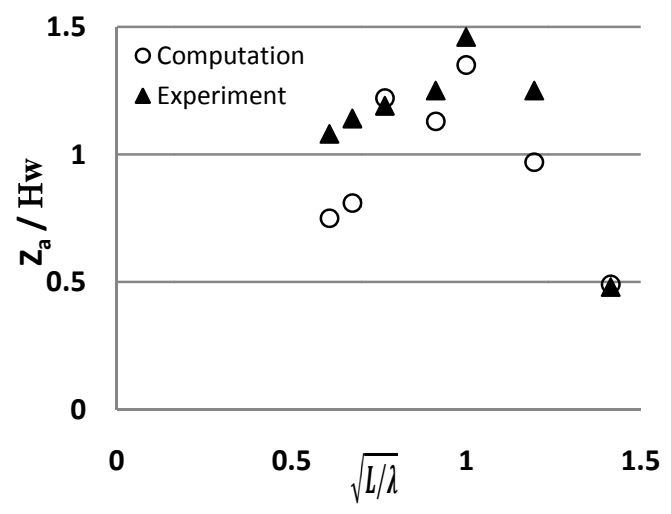

Fig. 5 Heave transfer function of the ship.

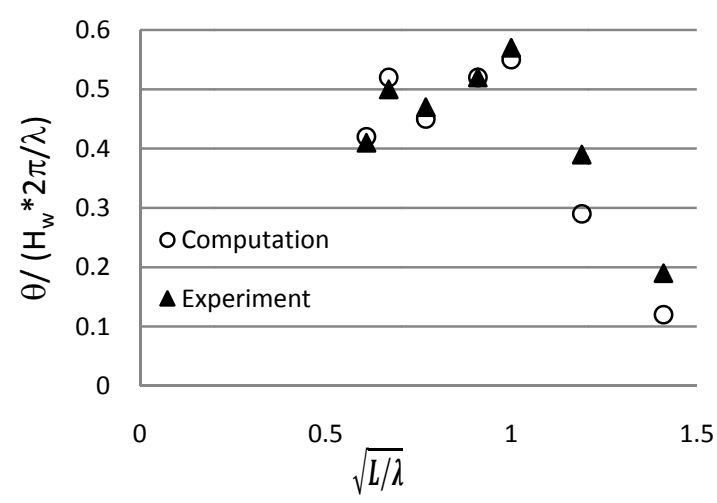

Fig. 6 Pitch transfer function of the ship. 
Based on transfer function of the heave and pitch, the motions were yielded maximum value when $\sqrt{L / \lambda}$ $=1$. Therefore, the numerical results show in good agreement with the experimental results.

\section{Results and Discussions}

\subsection{Resistance and Added Wave Resistance Using} Numerical Method

The computational conditions were set where the grid size is normalized by length between perpendiculars $\left(L_{p p}\right)$ 0.0025-0.015 $L_{p p}$ and the radius of free surface particle is $0.00125-0.005 L_{p p}$. Then, the radius of the $\mathrm{SPH}$ particle is $0.00125 L_{p p}$. In the model, the ferry is represented by a large number of the SPH particles traced by particle based method as shown in Fig. 7. For wave conditions, the incident regular wave height is set to $H_{w} / L_{p p}=0.01$. The wave length is ranged from $\lambda / L_{p p}=0.5$ to 2.0. In additions, the speed is set in maximum $F n=0.29$.

Ship resistance and added wave resistance have been normalized. Non-dimensional resistance is defined by water density $\rho$, speed $V$, and wetted surface area $S$. Fig. 8 shows total resistance of the ferry. The total resistance consists of both frictional resistance and pressure resistance. The frictional resistance is calculated using density function $\phi_{I}$ considering a water line and coefficient of resistance for flat plate turbulent boundary layer based on Schoenherr formula. The tendency of the total resistance from $F r=0.12$ to 0.17 decreasesand then increases until increases until $F n=0.29$. This resistance tendency is the same with our previous results [7] where it had been validated with experimental result.

Fig. 9 shows the non-dimensional added wave resistance in wave height $H_{w} / L_{p p}=0.01$ and $F r=0.29$. The nondimensional added wave resistance $\Delta R /\left(\rho g\left(H_{w} / 2\right)^{2} B^{2} / L\right)$ is normalized by wave height $H_{w}$, ship length $L$ and ship width $B$. Using this method, the added wave resistance is obtained from computation resultsbetweenthe resistancein thecalm waterand that in

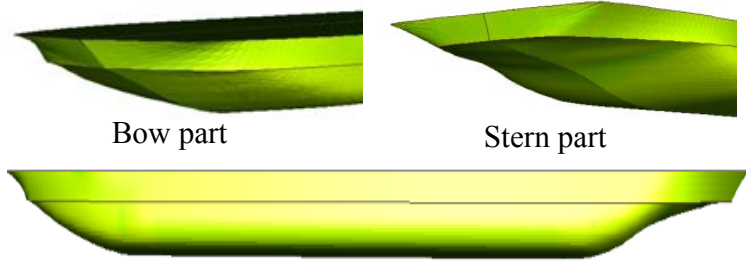

Fig. 7 The ferry model represented by a number of SPH particle.

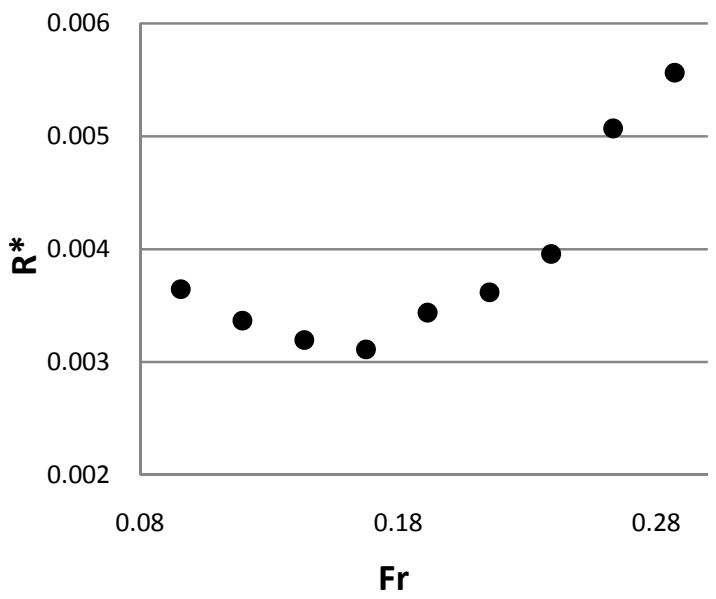

Fig. 8 The total resistance of the ferry.

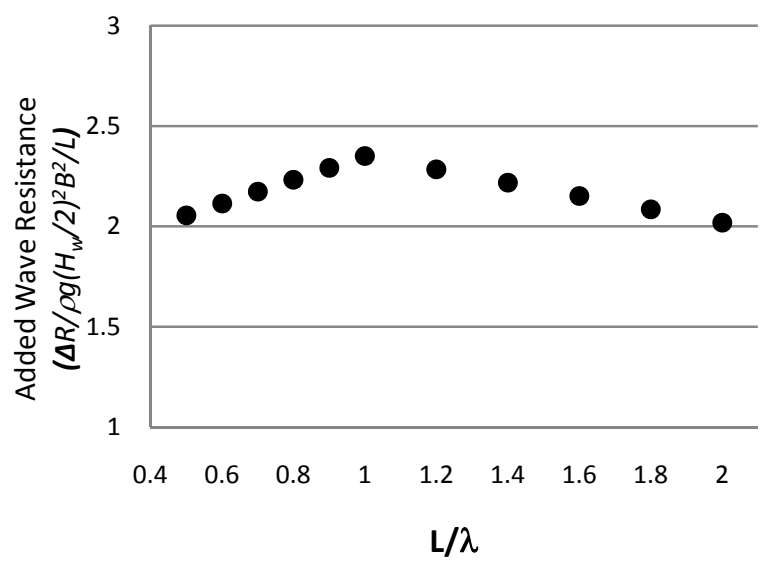

Fig. 9 The added wave resistance of the ferryin $H_{w} / L_{p p}=$ 0.01 .

the regular wave. The non-dimensional addedwave resistance decrease in increasing ship speed. The non-dimensional added wave resistance increases in increasing wave length $\lambda / L_{p p}=0.5$ to 1.0 in $F r=0.29$ and it then decreases after $\lambda / L_{p p}=1.0$. In addition, our method was applied also to that ferry with waveheight $H_{w} / L_{p p}=0.08$, where, this means extreme condition. The added wave resistance is higher than wave height $H_{w} / L_{p p}=0.01$. 
The numerical method was then applied to resistance of the ferry after modification its hull under still water. Bow and stern parts were only considered to be modified simultaneously. Figs. 10 and 11 are show an example bow and stern part modification. The bow-stern modifications are limited by longitudinal centre buoyancy $L C B$ in range $-3 \%$ to $+3 \%$ of amidship to avoid trim conditions. This means also that the ferry volume is kept constant eventhought it is after modification. The modification of bow and stern sections was done by trial and error where the movement of buoyancy point in range $-3 \%$ to $+3 \%$ which has been explained previously. Therefore, the modification forms were resulted sevenforms; M1, M2, M3, M4, M5, M6 and M7, respectively.

Fig. 12 shows resistance reduction each modified form. Based on the Fig. 12, the M1 form has higher resistance reduction around $5.7 \%$ compared with other modifications. The displacement of LCB point is about $-2.9 \%$ from amidship. This means that the modification in bow part could reduce significantly resistance compared with in stern part. Based on the research result, this is indicated that the modification M1 can reduced more generation of wave making resistance through the bow of M1 comparing with others and it also has slightly better and finest bow.

The numerical method can be used in preliminary design stage to predict ship resistance and added wave resistance. In order to optimize ship hull form under still water based on resistance and added wave resistance during initial design process, it could be useful as well.

\subsection{Prediction on Ship Motions by Conducting Experiment}

The seakeeping performance of a ship in preliminary design process is sometimes neglected. It is an important consideration to obtain proper design. Nowadays, there are some numerical methods available that could be used. To make more detail interpretation concerning on ship seakeeping per for mance and then develope our numerical method, we need more experimental results. Therefore, in section 5.2 the motions of ferry which has higher resistance

(a). Bow section

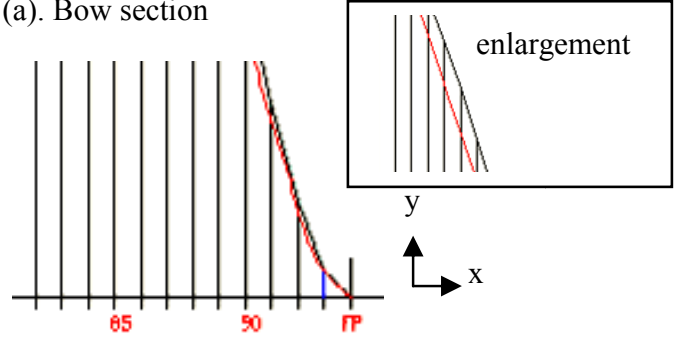

(b). Stern section

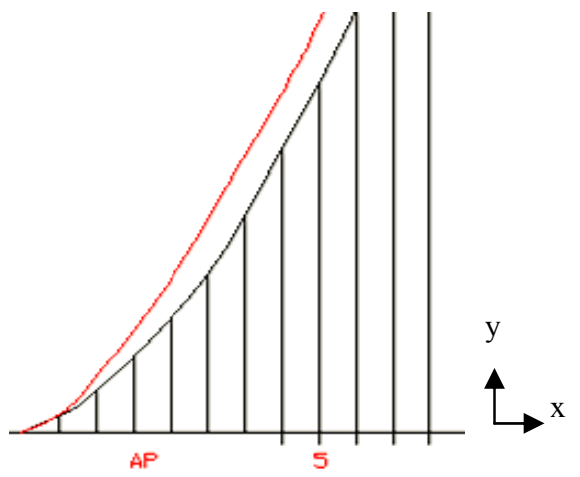

Fig. 10 Bow and stern sections area of the ferry.

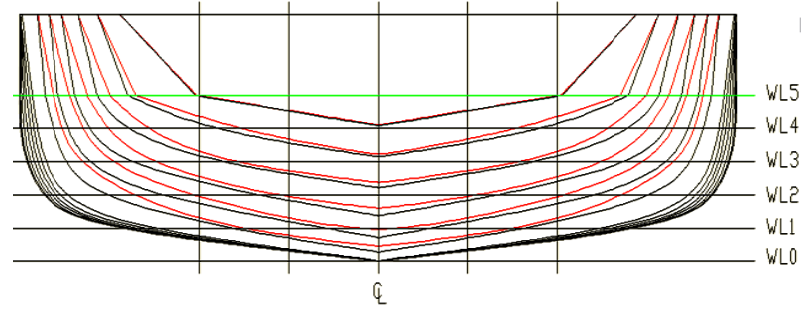

Fig. 11 Body lines form amidship to bow part both basic and modification forms.

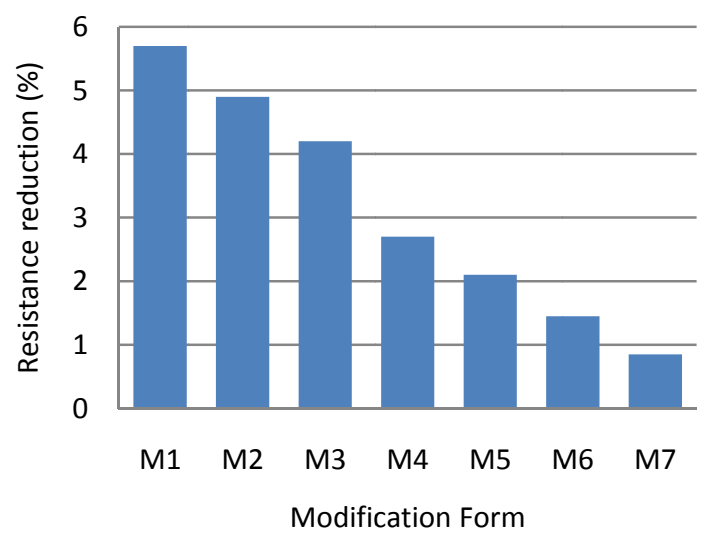

Fig. 12 The resistance reduction of each modified form. 

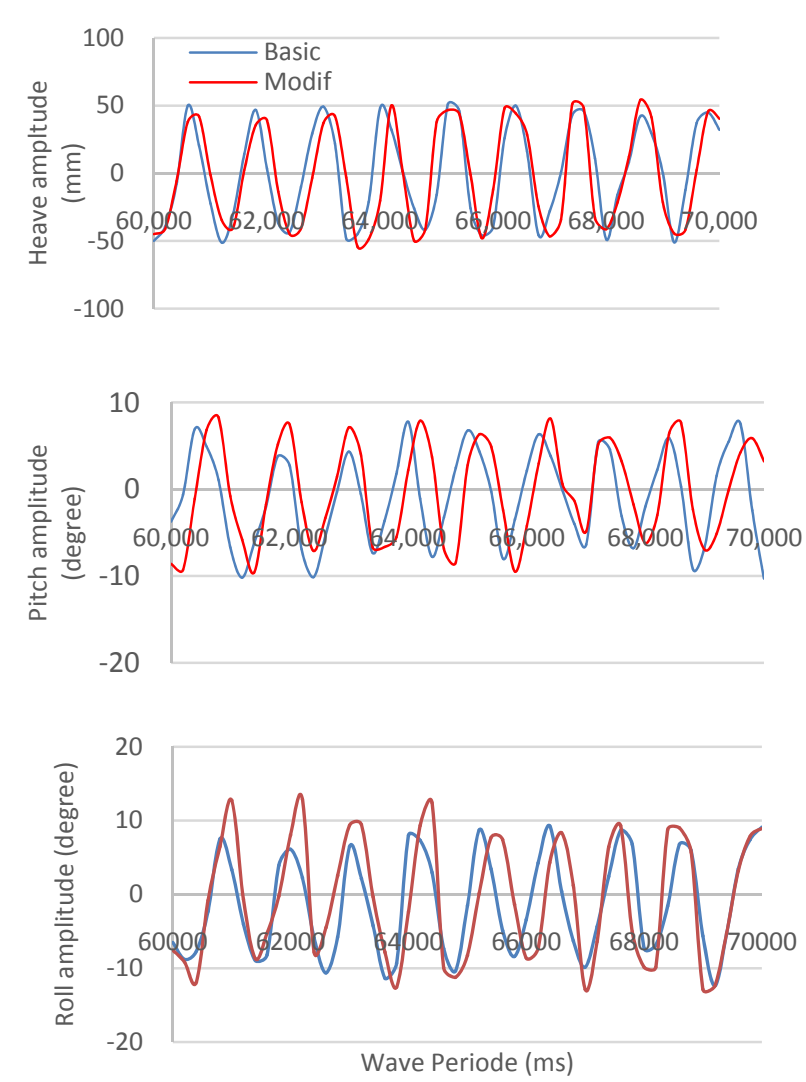

Fig. 13 Time history of heave, pitch, and roll amplitudes both basic andd modification forms.

reduction M1 are then predicted by conducting experiment in order to interpret ship seakeeping for preliminary design purpose. The speed model was setto zero. The heave, pitch, and roll are considered and others are fixed. The ferry main dimensions have similar with previous computational work. The other experimental parameters have been stated previously into experimental set-up sub-section.

In interpreting the motions, heave and pitch motions have been normalized. Non-dimensional heave is defined by $H_{v} / H_{w}$ and then pitch and roll are defined by $\theta /\left(H_{w} K\right)$, where, $H_{v}$ and $\theta$ are heave and pitch motions amplitude, respectively, $H_{w}$ the incident wave height and $K$ the wave number.

Fig. 13 shows time histories of the heave, pitch, and roll amplitudes. Based on that figure, the average non-dimensional heave amplitudes of the basic form and the modification form are 0.44 and 0.41 , respectively. Moreover, the averaged non-dimensional pitch amplitudes of the basic form and the modification form are 0.93 and 0.97 , respectively. They are seen closer both basic form and modification form. However, the average non-dimensional roll amplitudes of the basic form and the modification form are sufficient different 1.15 and 1.38, respectively.

The heave motion for both forms is less than $H_{v} / H_{w}$ $=1.0$. Also the pitch motion for both form is $\theta /\left(H_{w} K\right)=1.0$. These means that ship forms have a good geometry even the wave length $\lambda / L$ is greater than 2.0. However, the roll motion is greater than $\theta /\left(H_{w} K\right)=1.0$. This motion is concern point for that wave condition.

\section{Conclusions}

The present study, the hybrid Eulerian scheme with Lagrangian particle method can be applied practically to predict ship resistance and added wave resistance in preliminary design stage. Moreover, it is useful also to optimize ship hull form under still water based on resistance and added wave resistance. Based on modification work, the optimum resistance reduction of the modified form under still water could be achieved approximately $5.7 \%$. The modification in bow part could reduce significantly resistance compared with in stern part.

In additions, the heave, pitch, and roll motions have been predicted as well by conducting experiment. The modified form affects approximately $9 \%$ on increasing quiet small heave, pitch, and roll motion. Therefore, numerical method involved simultaneously with experimental work is proper way in preliminary design stage.

\section{Acknowledgments}

This research was funded by the LP2M Hasanuddin University with contract No. 16187/UN4-42/PL.09/2014. The authors are sincerely grateful to Prof. Sudirman as the Head of LP2M Hasanuddin University. The author would like to 
extend my acknowledgments to Prof. Hidemi Mutsuda who has always been sincere and helpful in making understanding and supporting me, and to all authors for their help in completing the research report and this paper.

\section{References}

[1] Zhu, M., Yoshida O., and Miyata, H. 1993. "Verification of the ViscousFlow-Field Simulation for Practical Hull Forms by a Finite Volume Method."InProceedingsof the 6th Int Conf on Numerical Ship Hydrodynamics, 469-87.

[2] Stern, F., Paterson, E.G., and Tahara, Y. 1996. CFDSHIP-IOWA: Computational Fluid Dynamics Method for Surface-Ship Boundary Layers, Wakes, and Wave Fields.Issue 381 of IIHR report, Iowa Institute of Hydraulic Research, College of Engineering, the University of Iowa.

[3] Sato, Y., Miyata, H., and Sato, T. 1999. "CFD Simulation of 3-Dimensional Motion of a Ship in Waves: Application to an Advancing Ship in Regular Heading Waves."J Mar Sci Technology 4: 108-16.

[4] Hu, C., Faltinsen, O.M., and Kashiwagi, M. 2005. "3-D Numerical Simulation of Freely Moving Floating Bodyby CIPMethod." InProceedingsof 15th Int Offshore and Polar Eng Conf(ISOPE), 4: 674-9.

[5] Nishigaki, M., Sato, K., and Ishikawa, S. 2007. "Ship Design Technology with CFD-Improvement of Propulsive Performance."Mitsubishi Heavy Industries, Ltd. Technical Review, Vol. 44 No. 3 (Sep. 2007).

[6] Mutsuda, H., and Doi, Y. 2009. "Numerical Simulation of Dynamic Response of Structure Caused by Wave Impact Pressure Using an Eulerian Scheme with Lagrangian Particles."In Proceedings of the 28th International Conference on Ocean, Offshore and Arctic Engineering, 661-70.

[7] Baso, S., Mutsuda, H., Kurihara, T., Kurokawa, T., Doi, Y., and Shi, J. 2010. "An Eulerian Scheme with Lagrangian Particles for Evaluation of Seakeeping Performance of a Ship in Nonlinear Wave." International Journal of Offshore and Polar Engineering21(2): 103-10.

[8] Baso, S., Mutsuda, H., Kawakami, K., Hashihira, K., and Doi, Y. 2011. "Numerical Study on Nonlinear Hydroelastic and Hydrodynamic Effects on Floating Body Using Eulerian Scheme with Lagragian Particles.'In Proceeding of the 21st International Society Offshore and Polar Engineers Conference, 610-7.

[9] Mutsuda, H., Ishida, A., Baso, S., and Doi, Y. 2013. "Numerical Investigation of Resistance Reduction of Fishing Boat by Improving Stern Part."Advanced Shipping and Ocean Engineering 2(3): 77-83.

[10] Mutsuda, H., and Yasuda, T. 2000. "Numerical Simulation of Turbulent Air-Water Mixing Layer Within Surf-Zone." In Proceedings of the 27th Int Conf on Coastal Eng, 755-68.

[11] Takewaki, H., and Yabe, T. 1987. "Cubic-Interpolated Pseudo Particle (CIP) Method Application to Nonlinear or Multi-dimensional Problems."Journal of Computational Physics70: 355-72.

[12] Barraf, D. 1997. An Introduction to Physically Based Modeling: Rigid Body SimulationI-Unconstrained Rigid Body Dynamics. SIGGRAPH'97 course note, D3. 